\title{
Ley 20.418: historia de una ley por la dignidad de la mujer chilena
}

La ley 20.418 se promulgó el 18 de enero de 2010 y su reglamento el 20 de febrero de 2013, después de 37 meses de trámites. La herramienta que precisa el ámbito de aplicación de la ley por el Sector Salud en Chile está vigente y es indispensable que los especialistas en la Salud de la Mujer lo conozcan a cabalidad para contribuir con la protección de la población a su cuidado.

Sin embargo, es necesario hacer algunos alcances que ilustren el contexto en el cual se genera esta legislación. Todo se inicia en diciembre de 2000 cuando el Instituto de Salud Pública aprueba el registro de un fármaco con levonorgestrel sólo en empaque de dos comprimidos de $750 \mu \mathrm{g}$ cada una para el uso como Píldora Anticonceptiva de Emergencia (PAE) en el riesgo de un coito fecundante no protegido y hasta un máximo de 6 días post coital, razón por la cual se la nominó píldora del día después, término que se ha prestado para confusiones por la población en general. Pasaron 9 años antes que este fármaco pudiese tener una existencia legal en Chile.

Se inicia en el país una fuerte discusión por considerar que la PAE es un fármaco abortivo y por lo tanto atentaba contra el artículo 119 del Código de Derecho Sanitario según el cual: "No podrá ejecutarse ninguna acción cuyo fin sea provocar un aborto".

El tema es expuesto en el $20^{\circ}$ Juzgado Civil de Santiago por una ONG en 2000, llegando a la Corte Suprema en 2004, que no refrenda la prohibición de la venta emitida por la Corte de Apelaciones. Se produce una confusión entre nombres comerciales y contenidos hormonales. La ONG hace el reclamo ante la Contraloría General de la República por el decreto $\mathrm{N}^{\circ} 48$ del Ministerio de Salud de 2007 que aprueba las Normas Nacionales sobre Regulación de la Fertilidad (1) y que se publican en el Diario Oficial. Esto no tiene aprobación de la Contraloría.

En abril de 2008, 36 diputados solicitan la inconstitucionalidad del Decreto ante el Tribunal Constitucional (TC) por atentar en contra la Constitución. Este es rechazado por asuntos de formas y luego de rectificar el requerimiento entra en dis- cusión. A fines de 2008, este Tribunal acogió como inconstitucional la Anticoncepción Hormonal de Emergencia y se desestimó la solicitud de inconstitucionalidad para el Dispositivo Intrauterino, como para la consejería y anticoncepción en adolescentes, a excepción de lo referente a Anticoncepción Hormonal de Emergencia.

Durante todos estos años el tema estuvo en forma permanente en todos los medios de comunicación, tanto nacional como internacional, y se difundieron ampliamente todas las investigaciones básico-clínicas publicadas, en especial las desarrolladas en Chile y en colaboración con otros centros de investigación, donde destacaron los trabajos del Profesor Dr. Horacio Croxatto, quien presentó en el seno de la Sociedad Chilena de Obstetricia y Ginecología (SOCHOG) sus publicaciones (2).

Ocurrida esta determinación del TC de Chile, el Ejecutivo presentó un proyecto de ley al Legislativo sobre información, orientación y prestaciones en materia de regulación de la fertilidad. Tema que fue ampliamente discutido en ambas cámaras siendo su resultado la Ley 20.418, que se encuentra en la sección de crónica de esta Revista.

Cabe hacer algunas reflexiones de este desarrollo. Llama la atención que Chile, siendo el primer país Latinoamericano en incluir a nivel nacional las prestaciones de control de la fertilidad en el programa regular de atención materno infantil, en 19621964, es el último que lo discute y aprueba en el poder Legislativo, de toda la Región.

La medida a nivel nacional fue aplicada por la existencia de un Servicio Nacional de Salud casi de cobertura universal. Ya en 1962, el Director General del SNS Dr. Gustavo Fricke, crea la Comisión Asesora de Política de Regulación de la Fecundidad con los profesores de Salud Pública y Obstetricia y Ginecología. En 1964, el Presidente de la República Eduardo Frei Montalva, siendo Ministro de Salud el Dr. Francisco Mardones Restat, se formula la primera Política de Planificación Familiar, para disminuir la morbilidad y mortalidad por aborto que significaba: $2 / 5$ de la mortalidad materna, dos 
egresos hospitalarios de aborto por cada 2 nacidos vivos, $8 \%$ del total de egresos hospitalarios del país, ocupación del $20 \%$ de las camas de maternidad y casi $40 \%$ del total de sangre transfundida (3).

En Costa Rica en 1972, casi 10 años después se incorpora en la Caja Costarricense del Seguro Social, un programa de Planificación Familiar en las prestaciones Materno Infantiles (4). Posteriormente se sumaron muchos otros países de la región pero en un contexto de discusión político demográfica, y por lo tanto el tema fue de debate en los Legislativos como ocurrió en México, Panamá, Colombia, Panamá, Venezuela, Ecuador, Perú, Brasil, Uruguay, Paraguay, Bolivia, y Argentina en 2004 con la incorporación de las Obras Sociales al Programa Nacional de Planificación Familiar $(5,6)$.

La discusión en el Legislativo chileno ha sido a consecuencias de razones ideológicas por determinados métodos de regulación de la fecundidad, más que por la definición de una Política de Población del Estado de Chile. La tendencia de la fecundidad en Chile siempre ha sido menor al resto de los países latinoamericanos, en conjunto con Argentina y Uruguay.

Al analizar el Reglamento se observa que el Primero, Segundo y Tercer artículo estatuyen el derecho de toda persona a ser informada y orientada acerca de regulación de la fecundidad, de su vida sexual y afectiva y el Tercero expresa que los establecimientos asistenciales del sector salud deben entregar información completa y sin sesgo de todas las alternativas autorizadas de métodos para la regulación de la fecundidad, la prevención del embarazo en la adolescencia, de las infecciones de transmisión sexual y de la violencia sexual, incluyendo mención del grado y porcentaje de efectividad. Estos tres primeros artículos del Reglamento incluyen los tres primeros párrafos del Primer Artículo de la Ley 20.418.

Sin embargo, no se ha reglamentado el cuarto párrafo que depende del Ministerio de Educación, donde se estatuye la inclusión en la enseñanza Media de los establecimientos educacionales reconocidos por el estado de un Programa de Educación Sexual, de acuerdo con el proyecto educativo, convicciones y creencias de cada establecimiento escolar, en conjunto con el centro de padres y apoderados.

Obviamente que esta materia no es del Ministerio de Salud. Pero, muestra una incongruencia entre la Ley y el Reglamento por falta de participación del Sector de Educación. Esto es grave y debe ser reparado por los deberes que el Estado debe cumplir con su población, por ley de la República. Mientras esto no exista no habrá recursos para estos programas, especialmente en los establecimientos escolares más vulnerable, donde se origina la mayor proporción los embarazos, en especial de las adolescentes menores de 14 años que siguen en aumento.
El Cuarto y Quinto artículo del reglamento reafirman el derecho de la persona a elegir libremente el método de regulación de la fertilidad de acuerdo a sus creencia y formación, y que corresponde al Sistema Nacional de Servicios de Salud entregar a la población que requiera los métodos anticonceptivos aprobados en el país que se disponga, de acuerdo con la prescripción del profesional competente, sean estos hormonales, como no hormonales, tales como aquellos combinados con estrógenos y progestágenos, de progestágeno sólo, hormonales de emergencia y no hormonales, naturales o artificiales a excepción de aquellos cuyo objetivo o efecto directo sea provocar un aborto. Esto claramente obliga a los servicios públicos a disponer de los métodos anticonceptivos y tener al personal capacitado para su indicación o inserción.

El Sexto artículo del reglamento, describe que al tratarse de un método anticonceptivo de emergencia solicitado por una persona menor de 14 años, el facultativo o funcionario que corresponda, tanto en el sector público como privado, procederá a la entrega de dicho medicamento, debiendo informar de este hecho con posterioridad, a su padre, madre o adulto responsable que la menor señale, para lo cual consignará los datos que sean precisos para dar cumplimiento a esta obligación.

Esto claramente no queda al arbitrio de las autoridades comunales o de un determinado consultorio público o privado. La objeción de conciencia individual no es motivo para dejar a una/un paciente sin la atención adecuada y en el caso que ocurra, el profesional de la objeción, debe indicar quien lo reemplaza en estas circunstancias o donde se derivará a la paciente, con documentación por escrito y coordinando personalmente la atención de la paciente. Si no existe ninguna de estas posibilidades, debe asumir su responsabilidad profesional y comunicar posteriormente de su incompatibilidad a las jefaturas para que se administre adecuadamente la prestación a la cual las pacientes tienen derecho por ley (7).

El Séptimo artículo del reglamento estatuye la confidencialidad y privacidad de la persona sobre sus opciones y conductas sexuales. El Octavo y último artículo obliga a los profesionales poner a disposición del Ministerio Público, los antecedentes en los casos que fuese posible presumir la existencia de un delito sexual en la persona del solicitante o de la persona para quien se solicita el ejercicio de estos derechos.

Los profesionales chilenos en la especialidad han tenido una larga experiencia desde 1964, en estas materias de regulación de la fecundidad y con la ley 20.418 y con su reglamento se perfecciona el ejercicio de esta área y se mejora la posibilidad de cumplir con los derechos de pacientes y familiares, especialmente de adolescentes.

Sin embargo, no podemos olvidar que aún no 
se desarrollan en los programas de enseñanza de Medicina de Pregrado y Posgrado, en todas las Facultades de Medicina, en forma adecuada los contenidos en anticoncepción, o se siguen haciendo en forma sesgada y de acuerdo a determinadas ideologías, sin respetar el derecho legal de pacientes y familiares que serán atendidos por esos profesionales inadecuadamente formados.

\section{Profesor Dr. Ramiro Molina C.}

\section{REFERENCIAS}

1. Ministerio de Salud. Gobierno de Chile. Instituto Chileno de Medicina Reproductiva. Asociación Chilena de Protección de la Familia. Normas Nacionales sobre Regulación de la Fecundidad. Decreto Supremo 48/07. 3 febrero 2007. Segunda Edición, Noviembre 2008. Disponible en: http://www.minsal.gob.cl/portal/ url/item/795c63caff4ede9fe04001011f014bf2.pdf. Acceso el 21 de abril de 2013.

2. Federación Internacional de Ginecología y Obstetricia (FIGO). Consorcio Internacional sobre anticoncepción de Emergencia (ICEC). Mecanismo de Acción. ¿De qué modo las píldoras anticonceptivas de Emergencia de levonorgestrel (PAE de LNG) previenen el embarazo? Marzo 2011. Disponible en: www.emergencycontraception.orgwww.figo.org. Acceso el 21 de abril 2013.

3. Claudia Rojas Mira. Historia de la política de Planificación Familiar en Chile: Un caso paradigmático. Disponible en: http://www.debatefeminista.com/PDF/ Articulos/histor1201.pdf. Acceso el 22 abril 2013

4. Evaluación del Programa de Planificación Familiar de la Caja Costarricence de Seguro Social. CCSS 1974. A Carrasco S., R. Molina C. OIT/NNUU. Informe 1974.

5. La Revolución Demográfica. Consejo Nacional de Población de México. Mayo de 1975. Editor Lic. Carlos Payán. Impresora Hermida SA. Ciudad de México.

6. Programa Nacional de Salud Sexual y Procreación Responsable. Dirección Nacional de Salud Materno Infantil. Ministerio de Salud de la Nación. Buenos Aires, Febrero 2003.

7. Grupo Interdisciplinario de Bioética. Consideraciones sobre la Objeción de Conciencia. Bioética y Debat 2012;18(66):2-12. Disponible en: http://www.bioeticadebat.org/contenidos/PDF/BD66ESP.pdf. Acceso el 22 de abril 2013. 